\title{
Historia de la Geología en el Bicentenario de la Argentina
}

\section{Prólogo}

Este volumen incluye trabajos presentados en el marco del IV Congreso Argentino de Historia de la Geología (ivcahgeo), que tuvo lugar en el Museo de La Plata los días 15 y 16 de septiembre de 2016 y que se desarrolló bajo el lema "La Geología en el Bicentenario de la Argentina", como conmemoración del 200 aniversario de la Declaración de la Independencia el 9 de Julio de 1816, por la cual la Argentina se convirtió en una República independiente.

El congreso tuvo aproximadamente 60 participantes y durante las sesiones hubo in total de 43 presentaciones, cuyos resúmenes fueron publicados en un Suplemento de la Revista del Museo de La Plata. Muchas de estas contribuciones fueron presentadas para su publicación, lo que ha permitido editar el presente Número Especial de la Revista del Museo de La Plata.

Estos trabajos incluyen estudios sobre contribuciones geológicas por parte de personalidades e instituciones y/o sobre áreas específicas, ya sea temática o geográfica. Las dedicadas a determinadas personalidades históricas, enfatizan en un contexto biográfico las contribuciones geológicas de determinados exploradores/naturalistas/geólogos/paleontólogos, i.e. G. Bonarelli y el mapa geoagrológico de Corrientes (por F.G. Aceñolaza), Luis de la Cruz y Goyeneche y los primero fósiles mencionados del centro-oeste de le Argentina (por B. Aguirre Urreta \& V.A. Ramos), Johannes Brüggen y sus aportes al desarrollo del conocimiento geológico de Chile (por R. Charrier, F. Hervé y P. Aceituno), I. Domeyko y la geología y la ciencia en Chile (por F. Hervé y R. Charrier), José M. Sobral y sus exploraciones petroleras en la Cuenca del Nirihuau (por E.G. Ottone), Handel T. Martin y los propósitos y resultados de su expedición paleontológica de 1903-1904 a la Formación Santa Cruz en la Patagonia austral (por S.F. Vizcaíno, P.D. Brinkman y R.F. Kay), Enrique Sparn y su aporte a la bibliografía geológica (por E. del V. Silva y R.N. Alonso), las contribuciones geológicas de Carlos F. Stubbe (por R.N. Alonso, N.G. Solís y E. del V. Silva), Roberto Caminos (por E.J. Llambias, C.A. Cingolani y A.M. Sato), E. Fossa Mancini (por A.C. Riccardi), y M.E. Teruggi (por L.A. Spalletti),

La historia de instituciones geológicas es considerada en lo que hace a, la historia de la geología en Uruguay (por J. Bossi), las contribuciones de naturalistas suizos vinculados al Museo de La Plata (por S.I. Carrasquero), las actividades del Museo de La Plata entre 1906 y 1966 (por A.C. Riccardi), el comienzo y desarrollo de la geología en la Universidad de Buenos Aires (por V.A. Ramos) y su reorganización después de 1955 (por R.J. Cucchi).

Aspectos históricos de campos y/o temas específicos son descriptos o discutidos en relación a: las ideas de Charles Darwin sobre el relleno sedimentario de valles andinos (por J.F. Mescua), la peleontología de Vertebrados en Argentina (por E. Ortíz-Jaureguizar, P. Posadas, S. Miguel, M.A. Abello, A.M. Luy, M. Hidalgo y E. Stubbs), la presentación de trabajos paleontológicos en 1910, durante el "American International Scientific Congress" (por A.R. Prieto), las contribuciones de los primeros geólogos petroleros de YPF en la región de Comodoro Rivadavia (por J.J. Hechem), M. Doello Jurado y la historia del meteorito El Toba (por J.M. Selles-Martínez), la exploración petrolera en la región central de la provincia de Santa Fe (por R. Calegari y S. Reinante), la publicación de la revista del Centro de Estudiantes de Ciencias Naturales en la Universidad de Buenos Aires (por R.J. Cucchi), la confección de cartas geológico-militares en la región de la Mesopotamia argentina (por F.G. Aceñolaza), A.V. Borrello y la historia de la geocronología Rb-Sr en La Plata (por C.A. Cingolani), la participación de mujeres en la paleontología argentina desde 1947 (por R. Herbst y L.M. Anzótegui). 


\title{
IV CONGRESO ARGENTINO DE HISTORIA DE LA GEOLOGÍA
}

\author{
- IVCAHGEO -
}

\section{Comisión Científica Honoraria}

Dr. Florencio G. Aceñolaza (Universidad Nacional de Tucumán), Dr. Guillermo L. Albanesi (Universidad Nacional de Córdoba), Dr. Ricardo N. Alonso (Universidad Nacional de Salta), Dr. Francisco Hervé (Universidad de Chile), Dr. Eduardo G. Ottone y Dr. Víctor A. Ramos (Universidad de Buenos Aires).

\section{Comisión Organizadora}

Dr. Alberto C. Riccardi (Presidente), Dr. Carlos A. Cingolani, Dr. Eduardo P. Tonni, Dr. Sergio F. Vizcaíno. Asistente: Lic. Andrea Bidone. Diseñador gráfico: Sr. Mario Campaña.

\section{Instituciones Auspiciantes}

Facultad de Ciencias Naturales y Museo (UNLP), Asociación Geológica Argentina, Asociación Paleontológica Argentina, Asociación Argentina de Sedimentología, Fundación Museo de La Plata "Francisco P. Moreno".

\section{Financiación}

Consejo Nacional de Investigaciones Científicas y Técnicas (CONICET), Universidad Nacional de La Plata (UNLP).

\section{Apoyo administrativo y logístico}

Fundación Museo de La Plata "Francisco P. Moreno", Museo de La Plata.

\section{Arbitrajes científicos}

F.G. Aceñolaza, G.L. Albanesi, R.N. Alonso, C.A. Cingolani, U. Cordani, E. Godoy, H.A. Leanza, E.G. Ottone, V.A. Ramos, A.C. Riccardi, E.P. Tonni, S. Vizcaino.

\section{CONGRESOS ANTERIORES}

I Congreso Argentino de Historia de la Geología (ICAHGEO), 20-22 de septiembre de 2007, San Miguel de Tucumán (Presidente: Dr. F.G. Aceñolaza). Trabajos publicados en: Aceñolaza, F.G., ed., 2008, Los Geólogos y la Geología en la Historia Argentina, INSUGEO Serie Correlación Geológica 16.

II Congreso Argentino de Historia de la Geología (IICAHGEO), 12-13 de agosto de 2010, Buenos Aires (Presidente: Dr. G.E. Ottone), Trabajos publicados en: Ottone, E.G., ed., 2011, Historia de la Geología, Revista de la Asociación Geológica Argentina 68, 3.

III Congreso Argentino de Historia de la Geología (IIICAHGEO), 21-31 de agosto de 2013, Salta (Presidente: Dr. R.N. Alonso). Trabajos publicados en: Alonso, R.N., ed., 2013, Actas III Congreso Argentino de Historia de la Geología - IIICAHGEO -, Mundo Gráfico Salta Editorial. 